\title{
A STEEPEST DESCENT METHOD FOR OSCILLATORY RIEMANN-HILBERT PROBLEMS
}

\section{P. DEIFT AND X. ZHOU}

In this announcement we present a general and new approach to analyzing the asymptotics of oscillatory Riemann-Hilbert problems. Such problems arise, in particular, in evaluating the long-time behavior of nonlinear wave equations solvable by the inverse scattering method. We will restrict ourselves here exclusively to the modified Korteweg de Vries (MKdV) equation,

$$
\begin{gathered}
y_{t}-6 y^{2} y_{x}+y_{x x x}=0, \quad-\infty<x<\infty, t \geq 0, \\
y(x, t=0)=y_{0}(x),
\end{gathered}
$$

but it will be clear immediately to the reader with some experience in the field, that the method extends naturally and easily to the general class of wave equations solvable by the inverse scattering method, such as the $\mathrm{KdV}$, nonlinear Schrödinger (NLS), and Boussinesq equations, etc., and also to "integrable" ordinary differential equations such as the Painlevé transcendents.

As described, for example, in [IN] or [BC], the inverse scattering method for the MKdV equation leads to a Riemann-Hilbert factorization problem for a $2 \times 2$ matrix valued function $m=m(\cdot ; x, t)$ analytic in $\mathbb{C} \backslash \mathbf{R}$,

$$
\left.\begin{array}{c}
m_{+}(z)=m_{-}(z) v_{x, t}, \quad z \in \mathbf{R}, \\
m(z) \rightarrow I \text { as } z \rightarrow \infty
\end{array}\right\}
$$

where

$$
\begin{gathered}
m_{ \pm}(z)=\lim _{\varepsilon \downarrow 0} m(z \pm i \varepsilon ; x, t), \\
v_{x, t}(z) \equiv e^{-i\left(4 t z^{3}+x z\right) \sigma_{3}} v(z) e^{i\left(4 t z^{3}+x z\right) \sigma_{3}}, \quad \sigma_{3}=\left(\begin{array}{cc}
1 & 0 \\
0 & -1
\end{array}\right),
\end{gathered}
$$

and

$$
v(z)=\left(\begin{array}{cc}
1-|r(z)|^{2} & -\overline{r(z)} \\
r(z) & 1
\end{array}\right)=\left(\begin{array}{cc}
1 & -\bar{r} \\
0 & 1
\end{array}\right)\left(\begin{array}{cc}
1 & 0 \\
r & 1
\end{array}\right) \equiv b_{-}^{-1} b_{+} .
$$

If $y_{0}(x)$ is in Schwartz space, then so is $r(z)$ and

$$
r(z)=-\overline{r(-z)}, \quad \sup _{z \in \mathbf{R}}|r(z)|<1 \text {. }
$$

From the inverse point of view, given $v(z)$, one considers a singular integral

Received by the editors February 13, 1991 and, in revised form, March 6, 1991.

1980 Mathematics Subject Classification (1985 Revision). Primary 35Q20; Secondary 35B40.

The work of the authors was supported in part by NSF Grants DMS-9001857 and DMS-9196033, respectively. 
equation (see [BC]) for the associated quantity $\mu(z ; x, t)=m_{+}(z ; x, t)\left(b_{+}^{-1}\right)_{x, t}$ $=m_{-}(z ; x, t)\left(b_{-}^{-1}\right)_{x, t}$ and the solution of the inverse problem is then given by

$$
y(x, t)=\left(\left[\sigma_{3}, \int_{\mathbf{R}} \mu(z ; x, t) w_{x, t}(z) \frac{d z}{2 \pi i}\right]\right)_{21}
$$

where

$$
w_{x, t}=\left(w_{+}\right)_{x, t}+\left(w_{-}\right)_{x, t}, \quad w_{ \pm}= \pm\left(b_{ \pm}-I\right) .
$$

Significant work on the long-time behavior of nonlinear wave equations solvable by the inverse scattering method, was first carried out by Manakov [M] and by Ablowitz and Newell [AN] in 1973. The decisive step was taken in 1976 when Zakharov and Manakov [ZM] were able to write down precise formulae, depending explicitly on the initial data, for the leading asymptotics for the $\mathrm{KdV}$, NLS, and sine-Gordon equations, in the physically interesting region $x=O(t)$. A complete description of the leading asymptotics of the solution of the Cauchy problem, with connection formulae between different asymptotic regions, was presented by Ablowitz and Segur [AS], but without precise information on the phase. The asymptotic formulae of Zakharov and Manakov were rigorously justified and extended to all orders by Buslaev and Sukhanov [BS 1-2] in the case of the KdV equation, and by Novokshenov [N] in the case of NLS.

The method of Zakharov and Manakov, pursued rigorously in [BS] and in [N], involves an ansatz for the asymptotic form of the solution and utilizes techniques that are somewhat removed from the classical framework of RiemannHilbert problems. In 1981, Its [I] returned to a method first proposed in 1973 by Manakov in [M], which was tied more closely to standard methods for the inverse problem. In [I] the Riemann-Hilbert problem was conjugated, up to small errors which decay as $t \rightarrow \infty$, by an appropriate parametrix, to a simpler Riemann-Hilbert problem, which in turn was solved explicitly by techniques from the theory of isomonodromic deformations. This technique provides a viable, and in principle, rigorous approach to the question of long-time asymptotics for a wide class of nonlinear wave equations (see [IN]). Finally we note that in [B], Buslaev derived asymptotic formulae for the KdV equation from an exact determinant formula for the solution of the inverse problem.

In our approach we consider the Riemann-Hilbert problem (1) directly, and by deforming contours in the spirit of the classical method of steepest descent, we show how to extract the leading asymptotics of the MKdV equation. In particular for $x<0$, let $\pm z_{0}= \pm \sqrt{|x| / 12 t}$ be the stationary phase points for $i\left(4 t z^{3}+x z\right)$. Then the first step in our method is to show that (1) can be deformed to a Riemann-Hilbert problem on a contour $\Sigma$ of shape (see Figure 1 ), in such a way that the jump matrices $v_{x, t}$ on $\mathbf{R} \subset \Sigma$ and on the compact part

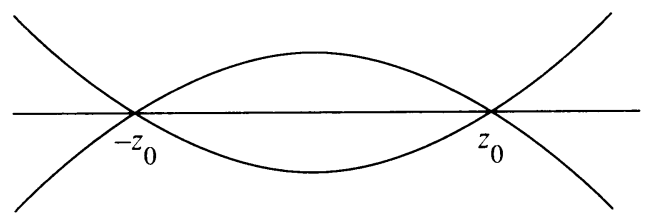

Figure 1 

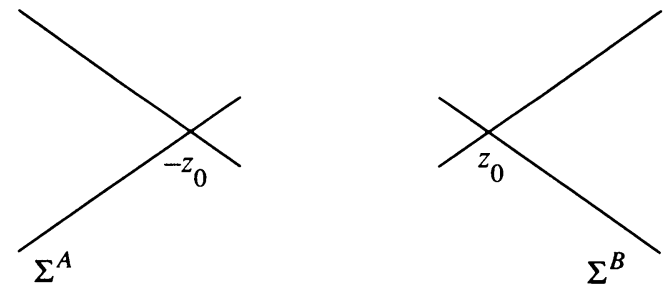

FIGURE 2

of $\Sigma \backslash \mathbf{R}$ away from $\pm z_{0}$, converge rapidly to the identity as $t \rightarrow \infty$. Thus we are left with a Riemann-Hilbert problem on a pair of crosses $\Sigma^{A} \cup \Sigma^{B}$ (see Figure 2). As $t \rightarrow \infty$, the interaction between $\Sigma^{A}$ and $\Sigma^{B}$ goes to zero to higher order and the contribution to $y(x, t)$ through (2) is simply the sum of the contributions from $\Sigma^{A}$ and $\Sigma^{B}$ separately. Under the scalings $z \rightarrow z\left(48 t z_{0}\right)^{-1 / 2} \mp z_{0}$, the problems on $\Sigma^{A}$ and $\Sigma^{B}$ are reduced to problems on a fixed cross, with jump matrices which are independent of time, and which can be solved explicitly in terms of parabolic cylinder functions, as in [I]. Substitution in (2) yields, finally, the asymptotics for $y(x, t)$.

Our result is the following: let

$$
\phi\left(z_{0}\right)=\arg \Gamma(i \nu)-\frac{\pi}{4}-\arg r\left(z_{0}\right)+\frac{1}{\pi} \int_{-z_{0}}^{z_{0}} \log \left|s-z_{0}\right| d\left(\log \left(1-|r(s)|^{2}\right)\right.
$$

(here $\Gamma$ is the standard gamma function) and let

$$
y_{a}=\left(\frac{\nu}{3 t z_{0}}\right)^{1 / 2} \cos \left(16 t z_{0}^{3}-\nu \log \left(192 t z_{0}^{3}\right)+\phi\left(z_{0}\right)\right),
$$

where $\nu=-(2 \pi)^{-1} \log \left(1-\left|r\left(z_{0}\right)\right|^{2}\right)>0$. Set $\tau=t z_{0}^{3}=\left(|x| / 12 t^{1 / 3}\right)^{3 / 2}$. Theorem. Let $y_{0}(x)$ lie in Schwartz space with reflection coefficient $r(z)$. As $t \rightarrow \infty$, the solution $y(x, t)$ of $\mathrm{MKdV}$ with initial data $y_{0}(x)$, has uniform leading asymptotics conveniently described at fixed $t \gg 1$, in the six regions shown in Figure 3.

$$
\begin{aligned}
& x<0 \quad x=0 \quad x>0
\end{aligned}
$$

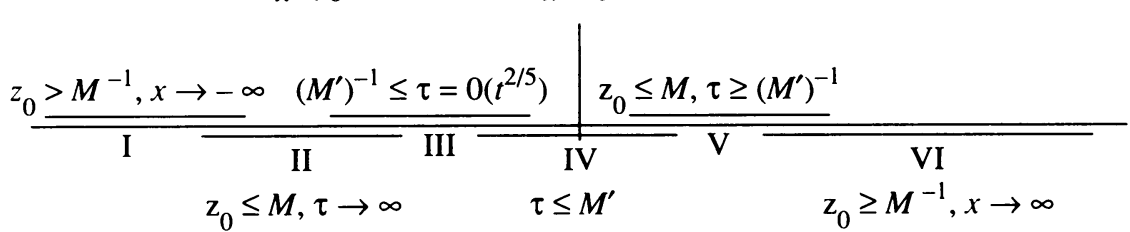

FIGURE 3

In region I, for any $j$,

$$
y(x, t)=y_{a}+O\left((-x)^{-j}+(-x)^{-3 / 4} C_{j}(-x / t)\right)
$$

where $C_{j}(\cdot)$ is rapidly decreasing. In region $\mathrm{II}$,

$$
y(x, t)=y_{a}+\left(t z_{0}\right)^{-1 / 2} O\left(\tau^{-1 / 4}\right) .
$$

In region $\mathrm{III}$,

$$
y(x, t)=(3 t)^{-1 / 3} p\left(x /(3 t)^{1 / 3}\right)+O\left(\tau^{2 / 3} / t^{2 / 3}\right),
$$

where $p$ is a Painlevé function of type II. 
In region $\mathrm{IV}$,

$$
y(x, t)=(3 t)^{-1 / 3} p\left(x /(3 t)^{1 / 3}\right)+O\left(t^{-2 / 3}\right) .
$$

In region $\mathrm{V}$, for any $j$,

$$
y(x, t)=(3 t)^{-1 / 3} p\left(x /(3 t)^{1 / 3}\right)+O\left(t^{-j}+t^{-2 / 3} e^{-12 \eta \tau^{2 / 3}}\right)
$$

for some $\eta>0$.

Finally, in region VI, for any $j$,

$$
y(x, t)=O\left((x+t)^{-j}\right) .
$$

Remark 1 . The reader may check that in the overlap regions the asymptotic forms do indeed match. Also the reader may check that in regions II and IV, the formulae for the leading asymptotics agree with those in [IN].

Remark 2. The above error estimates are not the best possible and in region II in particular, the $\tau^{-1 / 4}$ decay can certainly be improved.

Remark 3. There is no obstacle in the method to obtaining an asymptotic expansion for $y(x, t)$ to all orders.

Remark 4. As noted at the beginning of this announcement, the method we have presented extends naturally to the general class of nonlinear wave equations solvable by the inverse scattering method. Also, there is no difficulty in incorporating solutions with solitons.

\section{REFERENCES}

[AN] M. J. Ablowitz and A. C. Newell, The decay of the continuous spectrum for solutions of the Korteweg de Vries equation, J. Math. Phys. 14 (1973), 1277-1284.

[AS] M. J. Ablowitz and H. Segur, Asymptotic solutions of the Korteweg de Vries equation, Stud. Appl. Math. 57 (1977), 13-14.

[BC] R. Beals and R. Coifman, Scattering and inverse scattering for first order systems, Comm. Pure Appl. Math. 37 (1984), 39-90.

[B] V. S. Buslaev, Use of the determinant representation of solutions of the Korteweg de Vries equation for the investigation of their asymptotic behavior for large times, Uspekhi Mat. Nauk 34 (1981), 217-218.

[BS1] V. S. Buslaev and V. V. Sukhanov, Asymptotic behavior of solutions of the Korteweg de Vries equation, Proc. Sci. Seminar LOMI 120 (1982), 32-50. (Russian); transl. in J. Soviet Math. 34 (1986), 1905-1920.

[BS2] _ On the asymptotic behavior as $t \rightarrow \infty$ of the solutions of the equation $\psi_{x x}+u(x, t) \psi+$ $(\lambda / 4) \psi=0$ with potential $u$ satisfying the Korteweg de Vries equation, I, Prob. Math. Phys. 10 (1982), 70-102. (Russian); transl. in Selecta Math. Soviet 4, (1985), 225-248; II, Proc. Sci. Seminar LOMI 138 (1984), 8-32. (Russian); transl. in J. Soviet Math. 32 (1986), 426446; III, Prob. Math. Phys. (M. Birman, ed.) 11 (1986), 78-113. (Russian)

[I] A. R. Its, Asymptotics of solutions of the nonlinear Schrödinger equation and isomonodromic deformations of systems of linear differential equations, Soviet Math. Dokl. 24 (1981), 452456.

[IN] A. R. Its and V. Yu. Novokshenov, The isomonodromic deformation method in the theory of Painlevé equations, Lecture Notes in Math., vol. 1191, Springer-Verlag, Berlin and Heidelberg, 1986.

[M] S. V. Manakov, Nonlinear Fraunhofer diffraction, Zh. Èksper. Teoret. Fiz. 65 (1973), $1392-$ 1398. (Russian); transl. in Soviet Phys.-JETP, 38 (1974), 693-696. 
[N] V. Yu. Novokshenov, Asymptotics as $t \rightarrow \infty$ of the solution of the Cauchy problem for the nonlinear Schrödinger equation, Soviet Math. Dokl. 21 (1980), 529-533.

[ZM] V. E. Zakharov and S. V. Manakov, Asymptotic behavior of nonlinear wave systems integrated by the inverse method, Zh. Ėksper. Teoret. Fiz. 71 (1976), 203-215. (Russian); transl. in Sov. Phys.-JETP 44 (1976), 106-112.

Department of Mathematics, New York University-Courant Institute, New York, NEW YORK 10012

Department of Mathematics, Yale University, New Haven, Connecticut 06520 